\title{
Can microRNAs act as biomarkers of aging?
}

\author{
Luv Kashyap*
}

Division of Geriatric Medicine, Department of Medicine, University of Pittsburgh, $34715^{\text {th }}$ Ave., Suite 500, Pittsburgh, PA 15260v; Luv Kashyap Email: luvkashyap@gmail.com; *Corresponding author.

Received November 24, 2010; Accepted December 18, 2010; Published February 07, 2011

\begin{abstract}
:
Aging can be defined as a progressive decline in physiological efficiency regulated by an extremely complex multifactorial process. The genetic makeup of an individual appears to dictate this rate of aging in a species specific manner. For decades now, scientists have tried to look for tiny signatures or signs which might help us predict this rate of aging. MicroRNAs (miRNAs) are a unique class of short, non-coding RNAs that mediate the post-transcriptional regulation of gene expression ranging from developmental processes to disease induction or amelioration. Recently, they have also been implicated to have a role in aging in C.elegans. Based on the fact that there is a considerable similarity between aging in C.elegans and humans, these recent findings might suggest a possible role of miRNAs as bio-markers of aging. This mini-review brushes through the possibilities towards this direction.
\end{abstract}

Keywords: Aging, microRNAs, bio-markers, C.elegans.

\section{Background:}

MicroRNAs (miRNAs) are classes of small non-coding RNAs ( 22 nt) which normally function as negative regulators of target mRNA expression at the posttranscriptional level. They bind to the 3'UTR of target mRNAs through base pairing, resulting in target mRNAs cleavage or translational inhibition. It has also recently been demonstrated that miRNAs may function as positive regulators in some cases. It is estimated that $1-4 \%$ genes in the human genome are miRNAs and a single miRNA can regulate as many as 200 mRNAs [1].

The first discovered miRNA genes were lin-4 and let-7, which were identified in the tiny nematode model Caenorhabditis elegans [2-4]. A present, the functions of only a fraction of the identified miRNAs are known. In addition, multiple bioinformatics approaches have predicted target mRNA transcripts to suggest candidate genes regulated by miRNA interactions. Moreover, there is compiling evidence that suggesting that miRNAs might play critical roles in regulating key biological processes, such as cell growth, tissue differentiation, cell proliferation, embryonic development, and apoptosis $[\mathbf{1}, \mathbf{5}, \mathbf{6}]$.

At the present time, nothing is known about the role of miRNAs during aging in mammals, although the aging process has previously been shown to be associated with changes in the transcription of several protein coding genes $[7,8]$. In contrast, studies in the nematode worm C. elegans have shown that alterations in the expression of miRNAs are associated with aging [9]. For example, over-expression of lin-4 leads to extended lifespan, while loss of lin-4 resulted in a reduced lifespan in C.elegans [9]. In addition, another study reports an overall age-related decline in miRNA expression with aging in worms [10]. However, it is currently unclear how these findings translate to aging in vertebrate organisms in the context of both senescence and disease.

Can MicroRNAs act as biomarkers of aging?

C. elegans has been the premier model organism for studying the genetics of aging, and an excellent predictor of genes that also control mammalian aging [11]. Moreover, it is a highly suitable model for testing hypotheses regarding miRNA impact on aging biology because of its short lifespan and availability of exhaustive worm specific resources [11]. Instead, miRNAs can profoundly affect the expression of genes that do encode proteins by post-translational operation on the mRNA produced by such genes (Figure 1). The potential of miRNAs to influence aging has not been widely addressed, although recent studies have elegantly demonstrated how the developmental timing regulator miRNA lin-4 affects C. elegans lifespan [9]. Research in worms have shown that expression of various miRNAs change in abundance during adult life, suggesting significant potential to modulate health span and lifespan [10]. Indeed, miRNA lin-4 has been elegantly shown to influence lifespan and healthspan via its lin-14 mRNA target and the insulin signaling pathway [3].

Most of these C. elegans age-regulated miRNAs have sequence similarity with both fly and human miRNAs [13]. Understanding how they control the signaling networks that modulate aging in the worm should provide major insights into the aging process of mammalian species, including man. It is fascinating that miRNAs known as lin-4 and lin-14 have already been shown to regulate lifespan and developmental cues. Reducing the activity of lin- 4 shortened life span and accelerated tissue aging, whereas over expressing lin- 4 or reducing the activity of lin-14 extended life span. Lifespan extension conferred by a reduction in lin-14 was dependent on the DAF-16 and HSF-1 transcription factors, suggesting that the lin-4-lin-14 pair affects life span through the insulin/insulin-like growth factor-1 pathway. This work reveals a role for microRNAs and developmental timing genes in life-span regulation [13].

The question of course, is whether there are evolutionary-conserved homologs of lin-4 and lin-14 that control human longevity? A great many other miRNAs are similarly evolutionary conserved and are found in diverse species including worms, flies, zebrafish, mice and humans. It appears that when evolution finds a biological pathway that works for certain essential life functions in primitive species, when possible it carries these pathways forward with minimum required tinkering as it evolves higher species. This seems to be a general principle in genomics where many genes are the same in us and in yeast cells [14] 


\section{Bioinformation Volume 5}

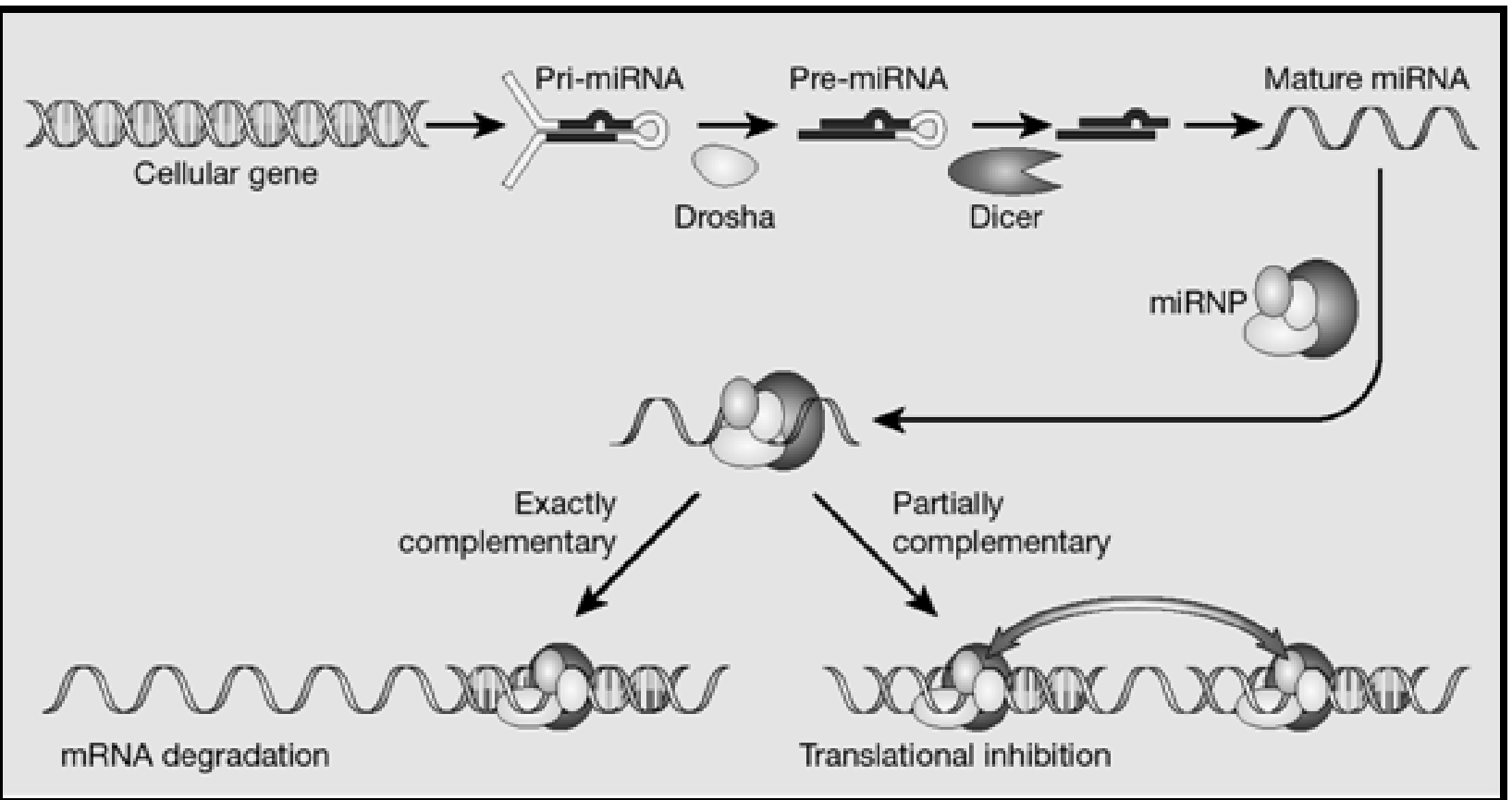

Figure 1: MicroRNAs mediated regulation of gene expression adapted from [12]. MicroRNA (miRNA), a group of short non-coding RNA that modulate the stability and translational efficiency of target messenger RNA via segments of dsRNA called dicer, which further get assembled into RISC, and are then targeted to mRNAs with perfect complementarity, and hence causing silencing by cleavage and degradation of the RNA or by formation of heterochromatin.

\section{Perspectives and speculations:}

Aging and death have fascinated philosophers and scientists for centuries. Aging is subject to regulation by genes that reside in pathways that have been conserved during evolution. The IGF- $1 / \mathrm{mTOR} / \mathrm{p} 53$ pathways are among those conserved pathways that impact upon longevity and agingrelated diseases [15]. Our understanding of how miRNAs work in humans is just getting off the ground. As indicated by recent research in field of microRNAs and aging, using worms as a model to investigate this correlation might not be a bad idea [13]. Let us fully exploit the fact that evolution carries biological machinery forward from species to species, including possibly machinery that controls aging. If we can thoroughly investigate various combinations of miRNAs already suspected or known to keep C. elegans young, and find homologs in humans of the same miRNAs, they may well keep us young too. We already know that the normal lifespans of nematodes of 20 days can be extended to 125 days by genetic manipulation [16, 17]. If that life extension factor of more than six could be translated to humans, it would mean normal lifespan of around 480 years.

\section{Acknowledgements:}

This work was published and supported by the Ellison Medical Foundation/AFAR Postdoctoral Fellows in Aging Research fellowship to Luv Kashyap.

\section{References:}

[1] A Esquela-Kerscher \& FJ Slack, Nat Rev Cancer 6 (4): 259 (2006) [PMID: 16557279]
[2] V Ambros \& HR Horvitz, Science 226 (4673): 409 (1984) [PMID: 6494891]

[3] RC Lee et al. Cell 75 (5): 843 (1993) [PMID: 8252621]

[4] BJ Reinhart et al. Nature 403 (6772): 901 (2000) [PMID: 10706289]

[5] M Lu et al. PLoS ONE 3 (10): e3420 (2008) [PMID: 18923704]

[6] R Roberts \& CJ Steer, J Cardiovasc Transl Res, 3 (3): 169 (2010) [PMID: 20560036]

[7] AK Roy et al. Ageing Res Rev 1(3): 367(2002) [PMID: 12067592]

[8] BT Weinert \& PS Timiras, J Appl Physiol 95(4): 1706 (2003) [PMID: 12970376]

[9] M Boehm \& F Slack, Science 310 (5756): 1954 (2005) [PMID 16373574]

[10] C Ibanez-Ventoso et al. Aging Cell 5 (3): 235 (2006) [PMID: 16842496]

[11] TE Johnson, Exp Gerontol 38 (11-12): 1329 (2003) [PMID: 14698813]

[12] CD Novina \& PA Sharp, Nature 430 (6996): 161 (2004) [PMID 15241403]

[13] C Ibanez-Ventoso \& M Driscoll, Curr Genomics 10 (3): 144 (2009) [PMID: 19881908]

[14] DE Bassett et al. Nat Genet 15 (4): 339 (1997) [PMID: 9090377]

[15] Z Feng et al. Cell Cycle 7(7): 842 (2008) [PMID: 18414039]

[16] JB Dorman et al. Genetics 141 (4): 1399 (1995) [PMID: 8601482]

[17] KD Kimura et al. Science 277 (5328): 942 (1997) [PMID: 9252323]

Edited by PN Pushparaj

Citation: Kashyap. Bioinformation 5(9): 396-397 (2011) License statement: This is an open-access article, which permits unrestricted use, distribution, and reproduction in any medium, for non-commercial purposes, provided the original author and source are credited 